\title{
The Promise of Multiwavelength and IFU Observations
}

\author{
Robert C. Kennicutt, Jr. ${ }^{1}$, Cai-Na Hao ${ }^{1}$, Benjamin D. Johnson ${ }^{1}$, \\ Fabian Rosales-Ortega, Angeles Díaz ${ }^{2}$, Anna Pasquale ${ }^{3}$ \& Sebastian \\ F. Sánchez ${ }^{4}$ \\ ${ }^{1}$ Institute of Astronomy, University of Cambridge, \\ Madingley Road, Cambridge, CB3 0HA, UK \\ ${ }^{2}$ Departamento de Física Teórica, C-XI, Universidad Autónoma de Madrid, \\ 28049 Madrid, Spain \\ ${ }^{3}$ Max-Planck Institute für Astronomie, Königstuhl 17, \\ 69117 Heidelberg, Germany \\ ${ }^{4}$ Centro Astronómico Hispano Alemám, Calar Alto, CSIC-MPG, \\ Jesús Durbán Remón 2, E-04004, Almeria, Spain
}

\begin{abstract}
Many of the advances of the last decade in this subject have been the result of large spectroscopic surveys of galaxies on the one hand, and multi-wavelength surveys of galaxies on the other. This talk highlights applications that exploit a combination of spectroscopic and multi-wavelength diagnostics. Most applications to date have been based on spatially integrated measurements of galaxies, but the introduction of wide-field integral field spectrometers promises to extend ths approach to spatially-resolved analyses of galaxies.
\end{abstract}

Keywords. dust, extinction, galaxies: ISM, galaxies: stellar content, infrared: galaxies, ultraviolet: galaxies, techniques: spectroscopic

\section{Introduction}

As this symposium has amply illustrated, many of the dramatic advances in our understanding of stellar populations in galaxies have resulted from the incredible growth of spectroscopic surveys such as SDSS, or from the flood of new multi-wavelength observations of galaxies, most notably from the Galaxy Evolution Explorer (GALEX) and the Spitzer Space Telescope. Recently attention has turned to exploiting the additional diagnostic power that comes from combining visible wavelength spectra with one or more observations outside of the visible window, and that is the topic we shall address here. Most current applications have focussed on integrated measurements of galaxies, but with the advent of wide-field integral field unit (IFU) spectrographs on many telescopes we can expect the approach to be extended to two-dimensional spatially resolved characterisation of nearby galaxies. We highlight examples of both in this talk. Since space does not allow for a thorough review of this subject, we highlight work being done at the IoA, and refer readers to other examples presented in this volume.

\section{Dust-Corrected Star Formation Rates of Galaxies}

One of the principal stellar population diagnostics in a galaxy spectrum is the presentday star formation rate (SFR). This can be readily estimated from the Balmer emissionline luminosity (Kennicutt 1998), but the results are subject to large and often uncertain corrections for dust attenuation. The dust correction can be measured from the Balmer 


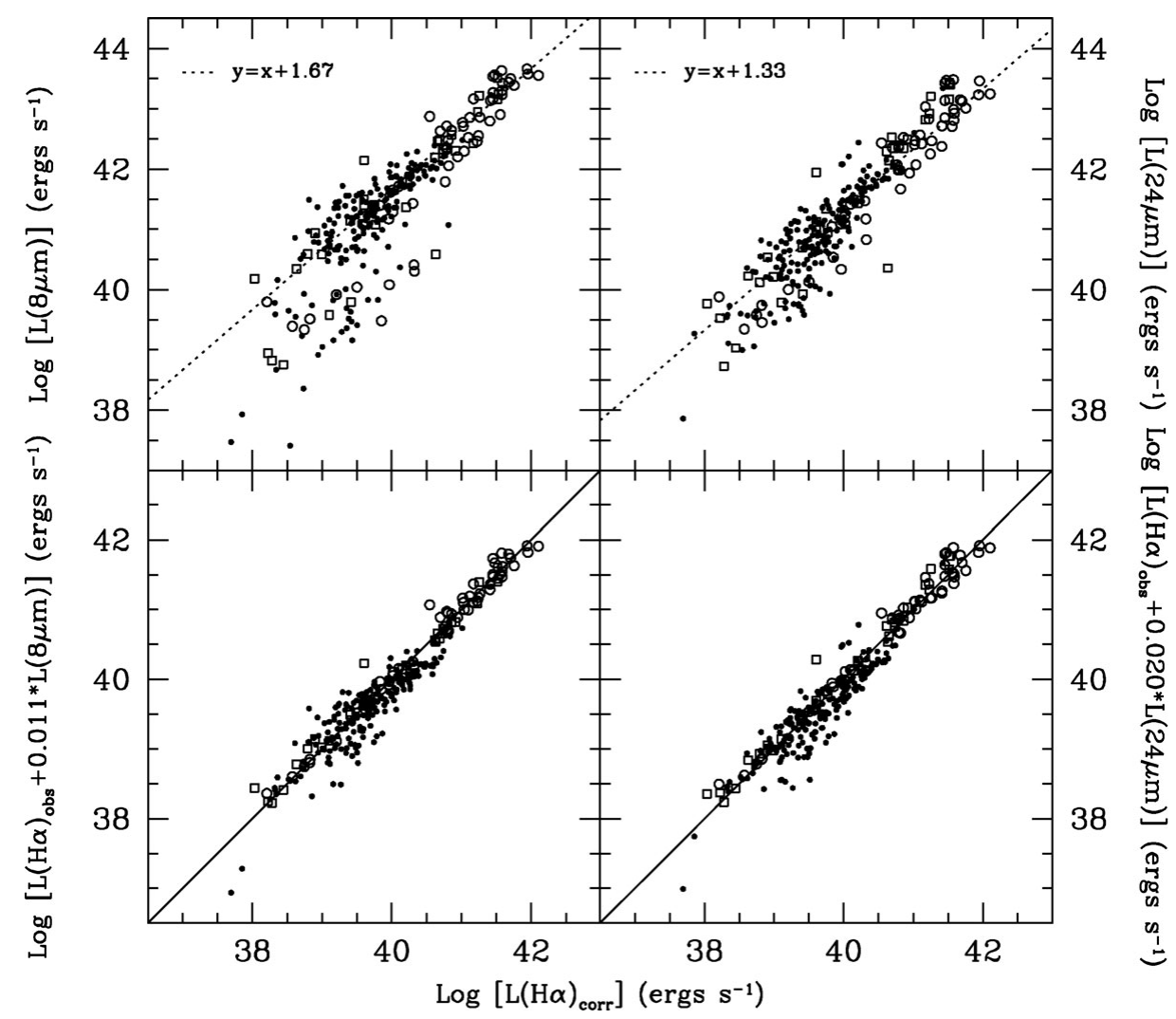

Figure 1. Top Panels: Comparison of $8 \mu \mathrm{m}$ (left) and $24 \mu \mathrm{m}$ (right) luminosities of galaxies with dust-corrected $\mathrm{H} \alpha$ luminosities of galaxies. Note the general correlations but the large dispersion and nonlinear trends, which arise from systematic variations in dust opacity and emissivity among the galaxies. Bottom Panels: Corresponding relation between linear combinations of observed H $\alpha$ plus IR fluxes. Figure taken from Kennicutt et al. (2009).

emission-line ratios, but this requires an integrated spectrum, high spectral resolution and signal/noise, and corrections for the often strong effects of stellar absorption, conditions that often are not met, especially for galaxies observed at large distances or redshifts.

A far more robust way to derive the dust attenuation is to combine the Balmer emission-line data with observations of the dust-reradiated infrared (IR) continuum. For star-forming galaxies the dust continuum luminosity roughly scales with the SFR (exclusive of any emission contributions from an AGN), and in principle it should be possible to calibrate the scaling factor between the IR luminosity and the SFR. Such IR-based SFR calibrations have been used extensively to measure the SFRs in dusty galaxies such as infrared-luminous starburst galaxies (see Kennicutt 1998 and references therein), but the infrared luminosity by itself does not provide an accurate SFR measure for normal galaxies, because it misses the substantial component of the young starlight that is not attenuated by dust.

The proper approach to addressing this problem is to construct composite SFR calibrations that are based on a combination of UV-visible SFR tracers (e.g., the $\mathrm{H} \alpha$ emission line), and a tracer of the dust-reradiated starlight, either the infrared luminosity or in principle the total radio continuum luminosity. Our group has explored this problem in depth (Kennicutt et al. 2009; Hao et al. 2009). In the most general case, reconstructing the unattenuated ionising flux or UV luminosity of a galaxy is a complex radiative 


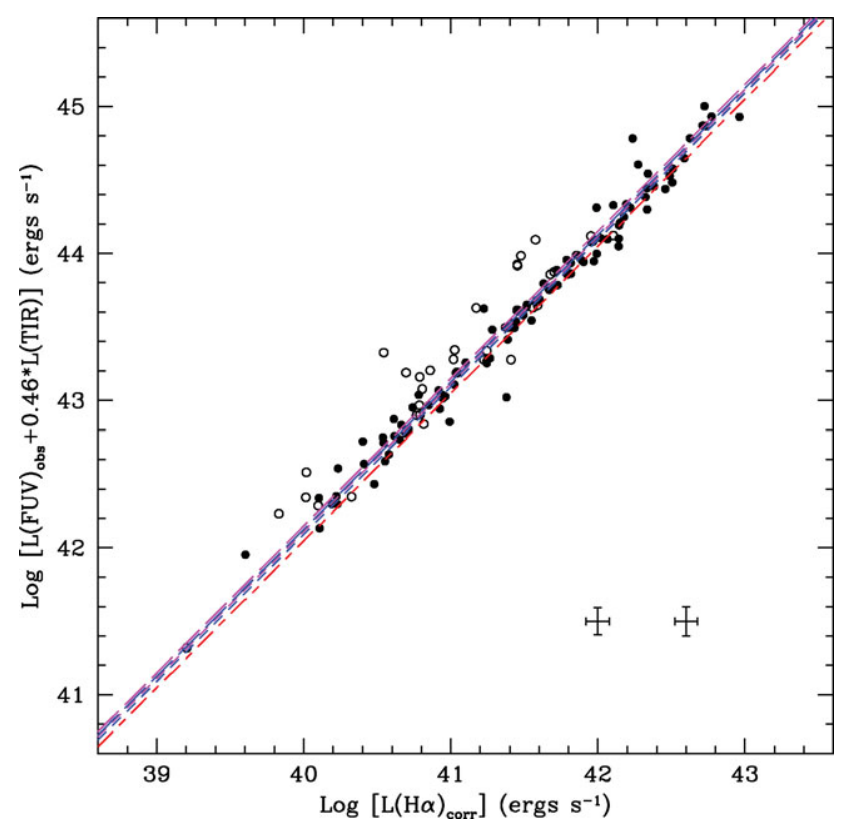

Figure 2. Relation between a linear combination of far-ultraviolet plus total infrared luminosities with the Balmer decrement corrected $\mathrm{H} \alpha$ luminosity for nearby galaxies. Figure taken from Hao et al. (2009, in preparation).

transfer problem. However it can be shown that when the typical dust opacity in the optical or UV tracer used is comparable to the mean opacity of a dust-absorbed photon, the dust attenuation can be reliably estimated from a linear combination of optical/UV and infrared/radio tracers (Kennicutt et al. 2009; Hao et al. 2009). We refer readers to these papers for details, and show examples here for how the approach is applied.

Figure 1 illustrates how the $\mathrm{H} \alpha$ luminosities of galaxies can be combined with their $8 \mu \mathrm{m}$ or $24 \mu \mathrm{m}$ dust luminosities to derive the attenuation-corrected $\mathrm{H} \alpha$ luminosity using galaxies with both integrated emission-line spectra (Moustakas \& Kennicutt 2006; Moustakas et al. 2009) and infrared photometry, from IRAS or the Spitzer Infrared Nearby Galaxies Survey (SINGS; Kennicutt et al. 2003). The top panels compare the observed $8 \mu \mathrm{m}$ (left) and $24 \mu \mathrm{m}$ (right) luminosities with the attenuation-corrected $\mathrm{H} \alpha$ luminosities, as derived from Balmer decrement corrected integrated spectra. There is a loose correlation between the two SFR estimators, but a large dispersion and non-linear trend that reflects the systematic increase in dust attenuation with increasing SFR and metallicity. The corresponding bottom panels show the relations between the Balmer-corrected $\mathrm{H} \alpha$ luminosities and a linear combination of (uncorrected) $\mathrm{H} \alpha$ and IR luminosities. These combinations were calibrated to match the Balmer-corrected values when averaged over the sample, so the agreement of mean SFR scales is by construction. However what is impressive is the low dispersion between the two attenuation estimates, and the complete removal of any nonlinear systematic difference in the SFR scales. We find that the tightest relations of all are found when one combines the observed $\mathrm{H} \alpha$ and total infrared luminosities (integrated over all IR wavelengths; TIR), with a dispersion of only $\pm 15 \%$ when compared to the Balmer decrement corrected luminosities or attenuations.

Similarly Figure 2, taken from Hao et al. (2009), shows the consistency between SFRs estimated from a linear combination of far-ultraviolet (FUV) and TIR luminosities, compared to the spectroscopically-derived $\mathrm{H} \alpha$-based SFRs. This FUV+TIR method is 
similar to that already introduced by many other authors (e.g., Meurer et al. 1999; Kong et al. 2004; Buat et al. 2005), but in this case is entirely empirically calibrated. In this case the consistency of the UV+IR based and $\mathrm{H} \alpha$ based calibrations is not a result of the calibration method but rather provides independent confirmation of the respective attenuation correction methods. Interestingly the results confirm that a simple $\mathrm{H} \alpha$ attenuation prescription based on the Balmer line ratios and a foreground screen dust model provides a reliable attenuation correction, confirming the earlier conclusions of Kewley et al. (2002).

As shown in Kennicutt et al. (2009) and Hao et al. (2009) this approach can be applied to calibrate a variety of other combinations of SFR tracers, for example any combination of FUV, NUV, $\mathrm{H} \alpha, \mathrm{H} \beta$, or [OII] $\lambda 3727$ index with the IR emission at 8 , 24, or $70 \mu \mathrm{m}$ or with the $1.4 \mathrm{GHz}$ radio continuum emission. These methods are not entirely free of systematic errors of their own. For example the methods assume isotropic extinction geometries which may break down in individual galaxies, and the dust emission remains somewhat sensitive to the stellar age mix. Our results suggeset that these effects are small in most galaxies, but can introduce systematic uncertainties of up to a factor of two in extreme cases. Nevertheless, these methods offer a severalfold improvement in the precision and systematic accuracy of dust attenuation corrections when compared to the most common prescriptions used previously.

\section{Breaking the Age vs Dust Degeneracy in Galaxy Spectra}

Dust attenuation can also inhibit the accurate age dating of stellar populations from their integrated continuum spectral energy distributions (SEDs) and spectra. This problem can be especially acute for measurements of star-forming galaxies observed at high redshift, where it is difficult to separate a downturn in flux in the blue from an ageing stellar population from a younger, dust-attenuated population.

In a series of recent papers Johnson et al. (2007a, 2007b, 2009) have combined SDSS spectra of nearby galaxies with continuum observations extending from the UV to the IR, to separate the independent effects of star formation history and dust attenuation on the observed SEDs of galaxies. The complementarity of the spectral and multi-wavelength information can be applied to the interpretation of both. For example the stellar population constraints provided by a measurement of the $D_{n}(4000)$ break in spectra and help to constrain the dust attenuation in the ultraviolet, while conversely the measurement of UV and IR colours can help to break the age vs dust degeneracy in the interpretation of the visible-wavelength data. Figure 3, which is taken from this work, illustrates one such application. It shows the $D_{n}(4000)$ values and NUV $(\sim 0.23 \mathrm{~nm})$ minus $3.6 \mu \mathrm{m}$ colors for a set of observed galaxies varying dust attenuation coded by color (from blue for low attenuation to red for high attenuation), along with model SEDs for unattenuated stellar populations (black points connnected by lines). The large wavelength baseline provided by the UV and IR measurements combined with the stellar population sensitivity of the $D_{n}(4000)$ index provides a clear separation of dust and age effects. This is only intended here as an illustration but it demonstrates the strong diagnostic synergy between the multiwavelength measurements and the optical spectra.

\section{Spatially Resolved Applications with IFUs}

There is tremendous potential in applying these types of techniques on a spatiallyresolved basis in galaxies, using multiwavelength maps of galaxies combined with spectra from IFU instruments. This capability is already being exploited by SAURON and other 

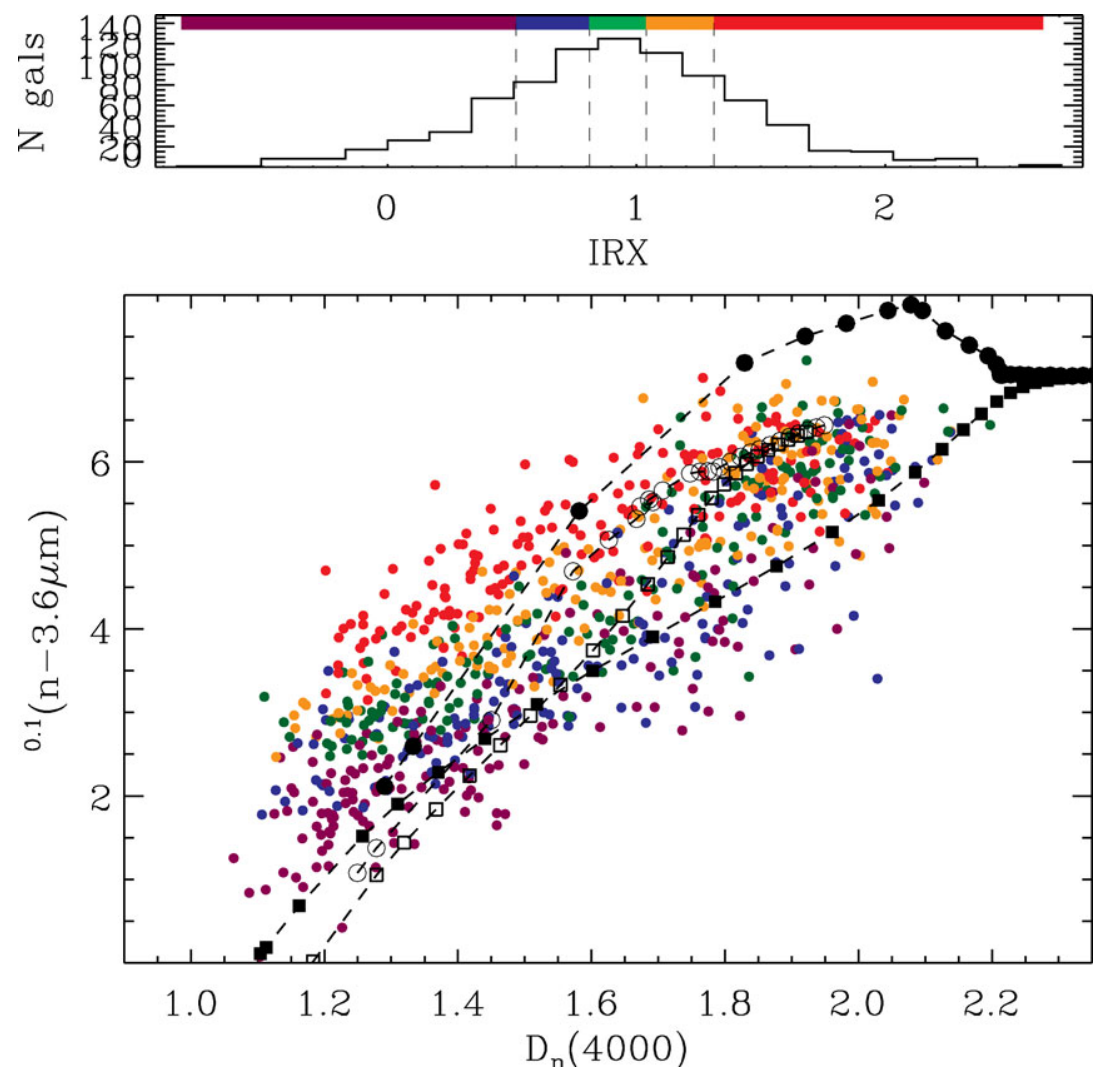

Figure 3. The lower panel shows the relation between near-ultraviolet minus $3.6 \mu \mathrm{m}$ color and Balmer discontinuity for galaxies with different dust attenuations, as indicated by the color coding. The corresponding attenuation scale is shown in the upper panel, in terms of IRX = $\log \left(L_{T I R} / L_{F U V}\right)$. Black points in the lower panel show the predictions of dust-free Bruzual and Charlot (2003) population synthesis models having exponentially declining SFR with e-folding times of $0.1 \mathrm{Gyr}$ (circles) and $1 \mathrm{Gyr}$ (squares) and metallicities of $0.4 Z_{\odot}$ (open) and $2.5 Z_{\odot}$ (filled). The upper The diagram illustrates the leverage provided by a combination of integrated spectra and UV - IR colors for breaking the degeneracy between age and dust attenuation.

projects, as evidenced by other papers presented at this symposium. Much of the previous work has concentrated on early-type galaxies, compact galaxies, or the circumnuclear regions of spiral galaxies, where the limited fields of view of most current instruments can be exploited most productively. However a new generation of IFU instruments, with spatial coverage of an arcminute or larger and spectral sensitivity extending into the blue (e.g., Kelz et al. 2006, Blanc et al. 2009), now make it possible to extend these surveys to larger galaxies, with good spatial matching to the $\sim 5$ arcsec resolution of the GALEX UV and Spitzer and Herschel IR maps.

Here we illustrate this emerging capability with results from the PPAK IFS Nearby Galaxies Survey (PINGS). The aims of this survey are to obtain spatially-complete maps of a sample of nearby galaxies, including galaxies from the SINGS survey, and apply them to study the gas-phase chemical abundances, star formation rates, and dust attenuation in two dimensions. The survey has been carried out using the PPAK IFU on the $3.5 \mathrm{~m}$ telescope of the Calar Alto Observatory. Figure 4, taken from Rosales-Ortega et al. (2009), shows the galaxies in the sample and the spatial coverage of the PPAK observations. 


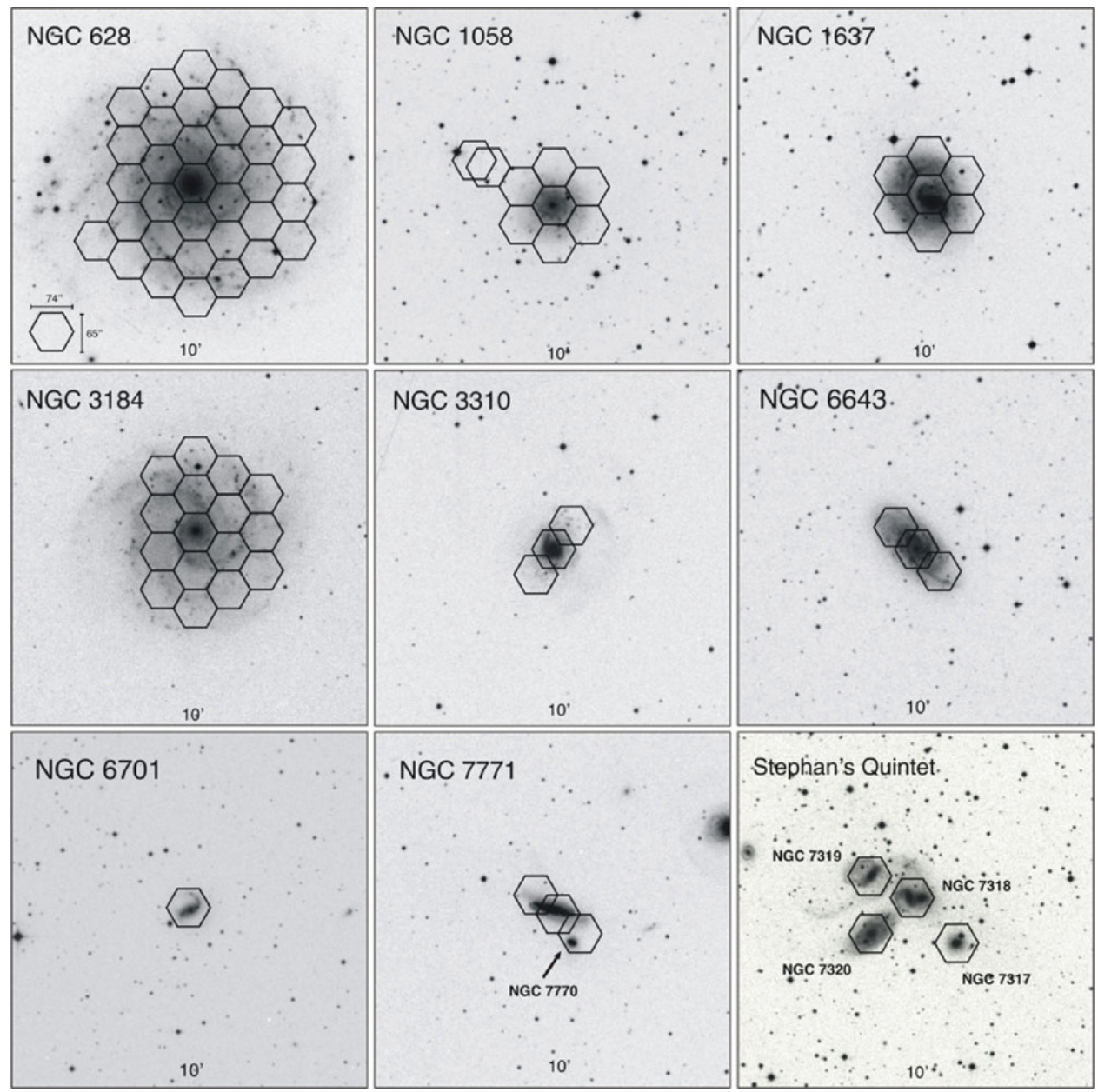

Figure 4. Galaxies in the PINGS IFU spectroscopic survey, with PPAK IFU positions indicated. Figure taken from Rosales-Ortega et al. (2009).

Figure 5, taken from the $\mathrm{PhD}$ dissertation of Fabian Rosales-Ortega (2009), shows maps of two abundance-sensitive strong-line ratios, the well-known $R_{23}$ parameter of Edmunds \& Pagel $(1984)([\mathrm{OII}]+[\mathrm{OIII}] / \mathrm{H} \beta)$ and the ratio $[\mathrm{NII}] /[\mathrm{OIII}]$ (Pagel \& Pettini (2004). The strong radial abundance gradient is seen, as well as the potential of such observations for testing for systematic distortions in the metallicity distribution, or for testing the reliability of these strong-line abundance tracers as a function of the physical conditions in the gas and the properties of the star-forming regions. The applications explored so far do not exploit the complementary capability provided by combining these IFU maps with multi-wavelength observations. However as part of the Herschel KINGFISH Key Programme we hope to use these spectra maps together with 0.15$250 \mu \mathrm{m}$ SED maps to apply the techniques described earlier to local measurements of dust attenuation, SFRs, and star formation histories in the discs of some of the PINGS galaxies.

This approach is also being extended with a new series of ambitious IFU surveys. A group in Texas led by Karl Gebhard is using the VIRUS-P instrument to carry out spectroscopic mapping of many of the SINGS galaxies (e.g, Blanc et al. 2009) and other 

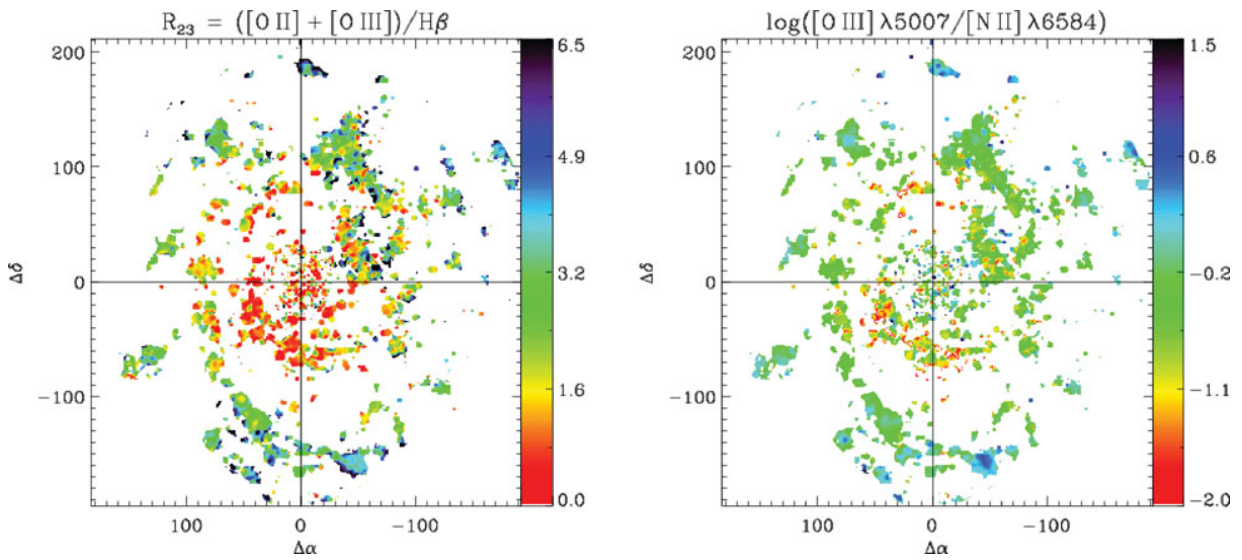

Figure 5. Contour maps of the abundance-sensitive emission-line ratios $R_{23}=$ $([\mathrm{OIII}]+[\mathrm{OII}]) / \mathrm{H} \beta$ (left) and $[\mathrm{OIII}] /[\mathrm{NII}]$ (right) for NGC 628, from a large mosaic of PPAK IFU pointings. Figure taken from Rosales-Ortega (2009).

types of nearby galaxies. The Calar Alto group has organised the CALIFA survey (still under review), which would carry out PPAK spectroscopy of a sample of a few hundred galaxies in the $\mathrm{z} \sim 0.02-0.03$ range, as a bridge between the single-fibre surveys of large surveys with SDSS and the more comprehensive mapping of very nearby galaxies with PINGS, VIRUS-P, and other surveys. Figure 6 shows a montage of galaxies observed as part of a pilot programme for CALIFA. The future of this subject is very bright, and it should bring important new insights into the star formation and stellar populations properties of the Hubble sequence, and the physical processes that form it.

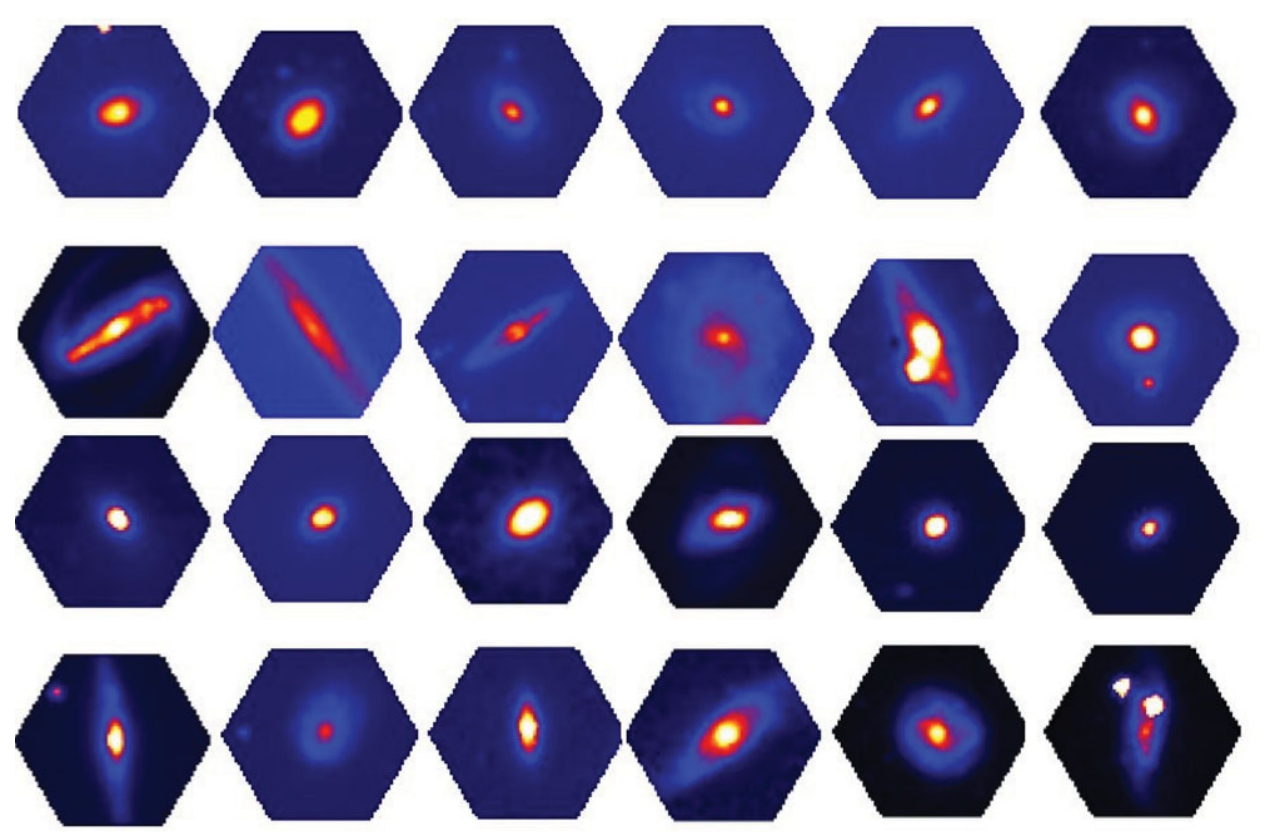

Figure 6. PPAK IFU observations of 24 nearby galaxies in a pilot study for the Calar Alto CALIFA survey. 


\section{References}

Blanc, G. A., Heiderman, A., Gebhardt, K., Evans, N. J., \& Adams, J. 2009, ApJ 704, 842

Bruzual, G. \& Charlot, S. 2003, MNRAS 344, 1000

Buat, V. et al. 2005, ApJ (Letters) 619, L51

Edmunds, M. G. \& Pagel, B. E. J. 1984, MNRAS 211, 507

Hao, C.-N. et al. 2009, in preparation

Kelz, A. et al. 2006, PASP 118, 129

Kewley, L. J., Geller, M. J., Jansen, R. A., \& Dopita, M. A. 2002, AJ, 124, 3135

Johnson, B. D. et al. 2007a, ApJS, 173, 377

Johnson, B. D. et al. 2007b, ApJS, 173, 392

Johnson, B. D. et al. 2009, in preparation

Kennicutt, R. C. $1988, A R A A 36,189$

Kennicutt, R. C. et al. 2003, PASP 115, 98

Kennicutt, R. C. et al. 2009, ApJ 703, 1672

Kong, X., Charlot, S., Brinchmann, J., \& Fall, S. M. 2004, MNRAS 349, 769

Meurer, G. R., Heckman, T. M., \& Calzetti, D. 1999, ApJ 521, 64

Moustakas, J. \& Kennicutt, R. C. 2006, ApJS, 164, 81

Pettini, M. \& Pagel, B. E. J. 2004, MNRAS 348, L59

Rosales-Ortega, F. 2009, PhD thesis, Univ Cambridge

Rosales-Ortega, F., Kennicutt, R. C., Sánchez, S. F., Díaz, A.I., Pasquali, A., Johnson, B. D., \& Hao, C. N. 2009, MNRAS, submitted 